\title{
Methodological approach to follow the effectiveness of a hand hygiene peer education training programme at Hungarian schools
}

\author{
HJ FEITH ${ }^{1 *}$, Á LEHOTSKY ${ }^{2}$, Á LUKÁCS ${ }^{1}$, E GRADVOHL $^{1}, \mathrm{R}_{\text {FÜZI }}^{3}, \mathrm{~S}_{\text {DARVAY MÉSZÁROSNÉ }}^{4}$, I KREKÓ BIHARINÉ $^{4}$, \\ ZS KARACS ${ }^{5}$, ZS KISS SOÓSNÉ ${ }^{6}$ and A FALUS ${ }^{7}$ \\ ${ }^{1}$ Faculty of Health Sciences, Department of Social Sciences, Semmelweis University, Budapest, Hungary \\ ${ }^{2}$ Hand-in-Scan Inc., Budapest, Hungary \\ ${ }^{3}$ Department of Public Health, Government Office of the Capital City Budapest, Budapest, Hungary \\ ${ }^{4}$ Faculty of Primary and Pre-School Education, Eötvös Lóránd University, Budapest, Hungary \\ ${ }^{5}$ Tamási Áron Elementary School and Bilingual German National Secondary School, Budapest, Hungary \\ ${ }^{6}$ Faculty of Health Sciences, Department of Clinical Studies, Semmelweis University, Budapest, Hungary \\ ${ }^{7}$ Faculty of Medicine, Department of Genetics, Cell- and Immunobiology, Semmelweis University, EDU VITAL Foundation, \\ Budapest, Hungary
}

(Received: September 12, 2017; revised manuscript received: October 11, 2017; accepted: February 20, 2018)

\begin{abstract}
Purpose: The authors intended to develop a novel procedure and research method that follows the effectiveness of the peer-educational approach in handwashing among school children. Materials and methods: To ask the children about their sociodemographic background, health behaviour, hand hygiene knowledge, and health attitudes, and questionnaires were applied. The education on proper handwashing procedures was followed by a test with a mobile UV-light detection system (Semmelweis Scanner, http://www.handinscan.com/), and the scans were evaluated through an intrinsic computer software. Results: Our newly developed questionnaire-based research method and the handrubbing technique followed by a test with a mobile UV-light detection system may become a reliable and valid scientific measurement of the effectiveness of hand hygiene training programmes. Conclusions: The Hand-in-Scan technology and questionnaire-based research method provide proper tools for evaluating the successful peer education method. It can significantly elevate the level of children's compliance, which leads to a better hygienic consciousness.
\end{abstract}

Keywords: peer education, health behaviour, hand hygiene, measurement-based research, schools, health promotion, health education

\section{INTRODUCTION}

There are many health promotion projects with peer education method for younger generations all around the world. Most programmes, however, seem to be descriptive and have not been evaluated systematically in a valid and reliable way [1-4].

The primary goals of the Study, Teach, Understand Health Promotion Programme (STAnD Programme - the Hungarian version of this programme is the "TANTUdSZ", the word is an acronym, which includes the beginning of the following words: Study, Teach, and Understand) is to teach children who are attending pre, primary, and secondary schools on how to a reach healthy lifestyle, a healthconscious behaviour and to measure the effectiveness of the training programmes as well as the impact of the health promotion interventions. The five targets of health promotion programmes of the STAnD project are personal hygiene, healthy diet, exercise, mental health, and first aid.

This concept not only includes knowledge transfer, but also internalizing the knowledge and becoming simultaneously exemplary, teachable, and attractive for youngsters.
The older generation students are involved in educating and shaping the attitudes of the younger ones. The basic principle of our special educational methodologies is underlined by several other surveys and implemented pedagogical practices [3, 5-8]. In younger age groups, few year older children and youngsters could serve as attractive "role models" to follow for the younger ones, while providing a kind of contemporary education $[9,10]$. A non-conscious, latent impact also exists in the case where the responsible behaviour of the person who transfers the knowledge appears as a model to be followed [11-14].

The STAnD Programme can be divided into two branches: a preparatory course and a peer education programme. The preparatory course is an intensive studentcentred higher education programme for healthcare and pedagogical university students by teachers of health and pedagogy sciences and an education programme for peer educators by students under the supporting supervision of

* Corresponding author: Helga Judit Feith; Faculty of Health Sciences, Department of Social Sciences, Semmelweis University, Vas utca 17, Budapest H-1088, Hungary; E-mail: feith@se-etk.hu

This is an open-access article distributed under the terms of the Creative Commons Attribution-NonCommercial 4.0 International License, which permits unrestricted use, distribution, and reproduction in any medium for non-commercial purposes, provided the original author and source are credited, a link to the CC License is provided, and changes - if any - are indicated. 
tutors. The peer education programme is a health education programme for children (aged 3-20 years) by students and peer educators under the supervision of tutors. Students and peer educators work together in a team (4-6 higher education students with 2-3 secondary school students) with tutorial support from universities. Tutors are responsible for implementing all interventions. Our peer education programmes are based on different target education levels: preschool (3-6 years), elementary school (7-14 years), and secondary school (15-20 years).

Hand hygiene interventions are frequently overlooked in preschools and schools, although these everyday protocols can be easily rehearsed and accomplished. This fact, emphasized in several health reports, is extremely unfortunate, since avoidance of many infectious diseases among other consequences may be able to radically decrease school absenteeism [15-17]. International sources call attention to these unpleasant circumstances and prompt the need for including and intensifying hand hygiene in education [18-23].

The primary aim of the STAnD Hand Washing Programme (SHWP) is to teach children on how to improve the hand hygiene technique and develop a health-conscious behaviour with the help of our peer education methodology. The SHWP consists of active, playful, and age-specific health education programmes that are based on a creative methodology under professional supervision. The most common teaching activities are games, simple science experiments, creative representations (e.g., drawing and painting and making models), drama activities, literature (e.g., listening or writing stories, poems, and song texts), and music (e.g., movement for music and singing songs).

In this study, we present the multidisciplinary research methodology of the hand hygiene peer education training programme and summarize our methodological trials using a fluorescence-based screening tool combined with a computerized evaluation of the handwashing procedure.

\section{MATERIALS AND METHODS}

\section{Sampling}

The target population of our hand hygiene training programme and research is formed from different target education levels: preschool (3-6 years), elementary school (7-10 and 10-14 years), and secondary school (15-20 years). Children can take part in our survey randomly as a participant of SHWP or as a member of the control group in the same age in the target schools. Our simple random sample of the SHWP selection is based on the alphabetical order of the children's names in their classes. This means that the second part of the class belongs to the SHWP and the first part of the class joins another health promotion programme. The control group, which is one class at every educational level, is also chosen randomly.

\section{Research instruments}

The SHWP intends to measure the effectiveness of the hand hygiene training programmes and the impact of the health promotion interventions with two research methods: (i) quantitative research with a newly developed and structured questionnaire and (ii) assessment of the hand-rubbing technique.

Quantitative social research. In our self-administered and self-structured questionnaire containing closed- and some open-ended questions with comparative and noncomparative scaling techniques, we use some items of various validated [24] and non-validated surveys [25], which we have complemented with our own questions and response scales. One of our important goals for the future is to validate our items. The questionnaire of the target population of SHWP study consists of the following six main dimensions.

1. Basic sociodemographic information about sex; age; number of siblings (living together); own room [24]; number of mobile phones, computers, cars, and bathrooms in the family [25]; number of good friends [24]; educational level of parents (only at secondary school students); and scholastic record of students with 5-level Likert item scale.

2. Self-perceived health status [24].

3. Level of knowledge about optimal length of handwashing, important tools of a correct handwashing (closed question with more correct answers), the best type of the hand-drying methods (closed question with only one correct answer), and the best type of the soap (closed question with only one correct answer).

4. Health behaviour in terms of handwashing habits with dichotomous survey questions (e.g., after using public transport; before/after eating and before/after using toilet; before sleeping; after touching pet; after arriving home; after playground; after painting, gluing, and drawing; and before watching TV) and ordinary length of handwashing (open-ended question).

5. Attitudes about handwashing, cleanness/uncleanness, and health with semantic differential scale questions (5-item scales - ranging from 1: no, I totally disagree to 5: yes, I totally agree - in age group 14-17 years; dichotomous answer in age group 6-10 years). In Table 1, a set of statements are shown, which focus on hygiene-related behaviour.

6. The level of satisfaction with the SHWP. Respondents are asked to evaluate different statements about the quality and effectiveness of the training programme with Likert item scales.

Physical measurement-based research. Assessment of hand hygiene technique by a fluorescein test is a widespread procedure that is based on the ultraviolet (UV) radiation of the distribution of fluorescein marker on the entire surface of the hand. However, earlier studies failed to show the quantitative distribution of the dye on the region of the hand covered by the indicator material.

A newly developed method called the Hand-in-Scan with the related software is used during the research for our measurements (Semmelweis Scanner, http://www.handinscan.com/). The device was developed by a team of engineers whose purpose was to create an objective control device for hand hygiene performance of both medical personnel and youngsters in various school programmes. Objectivity, which is crucial in efficiency assessment, is realized by quantitative computer evaluation of saved and 
Table 1. The attitude statements of the developed hand hygiene peer education questionnaire in the frame of Study, Teach, Understand Health Promotion Programme (STAnD Programme)

How much do you agree with the following statements?

It is important to lead a healthy lifestyle already in childhood.

Basic regular daily cleaning (bathing, handwashing, and tooth brushing) contributes to being more protected against illnesses.

Regular handwashing is not important, because we need some dirt to preserve our health.

One cannot do much to keep their health safe.

Just by washing my hands thoroughly, I do something to keep my health safe.

Properly cleaned and kept clean, we also protect the health of others.

As an adult, cleanliness is (will be) important for me in order to give a good example to kids/my kids.

One of the most important values in my life is my health.

I think regular daily washing (bathing, handwashing, and tooth brushing) is a waste of time.

stored images. Semmelweis Scanner is a mobile digital device that can perform the objective evaluation of the hand's disinfection ratio [24] and is suitable to teach children and youngsters to the culture of handwashing by direct feedback observation. This tool can be technically divided into three parts: a frame structure, a digital camera, and a central computer module. The general task of computerized image processing is digital imaging and image capture and further statistical processing by the analysis of captured images and hand regions. This new technology is applying a clustering algorithm with the aim of separating the clean and the dirty parts of the hand with both quantitative and qualitative assessments [26].

After the measurements, the UV-A radiation obtained from the Semmelweis Scanner device is evaluated retrospectively. The surface of the hand is divided into $9+1$ regions, including the palm and the side of the hand. The fingers either represent separate regions, or the fluorescence of fingers is evaluated as separate unified regions. This solution was chosen based on the observations obtained prior to this survey $[26,27]$.

\section{Data collecting procedure}

The immediate short-term effects and changes of the SHWP can be detected with the help of questionnaires and the assessment of hand-rubbing technique by UV-light-based imaging technology among the target population.

Our questionnaire focuses on the changes in the levels of knowledge, as well as health behaviour and attitude before and after the health education interventions. The assessment of the hand-rubbing technique by UV-light-based imaging technology, as described earlier, focuses on the coverage of the different regions of the hand by marker dye.

We make experimental groups and control groups randomly at every educational stage to measure the effectiveness of the preventive interventions. We pretest the members of experimental and control groups before the intervention. Next, we manipulate the independent variables using 4-or-8-lesson peer education programmes in the experimental groups and not using any teaching techniques for the control groups. After the interventions, we again test the members of the experimental groups to compare the effectiveness of preventive interventions between the groups by sex, socioeconomic status, educational stage, prevention topic, and length of intervention. Furthermore, we measure the efficiency of the handwashing procedures of the students, as described above.

Our programme also examines the long-term effects of the SHWP, so the members of experimental and control groups are retested in 3-4 months. The aim is to detect whether the hand hygiene peer education training

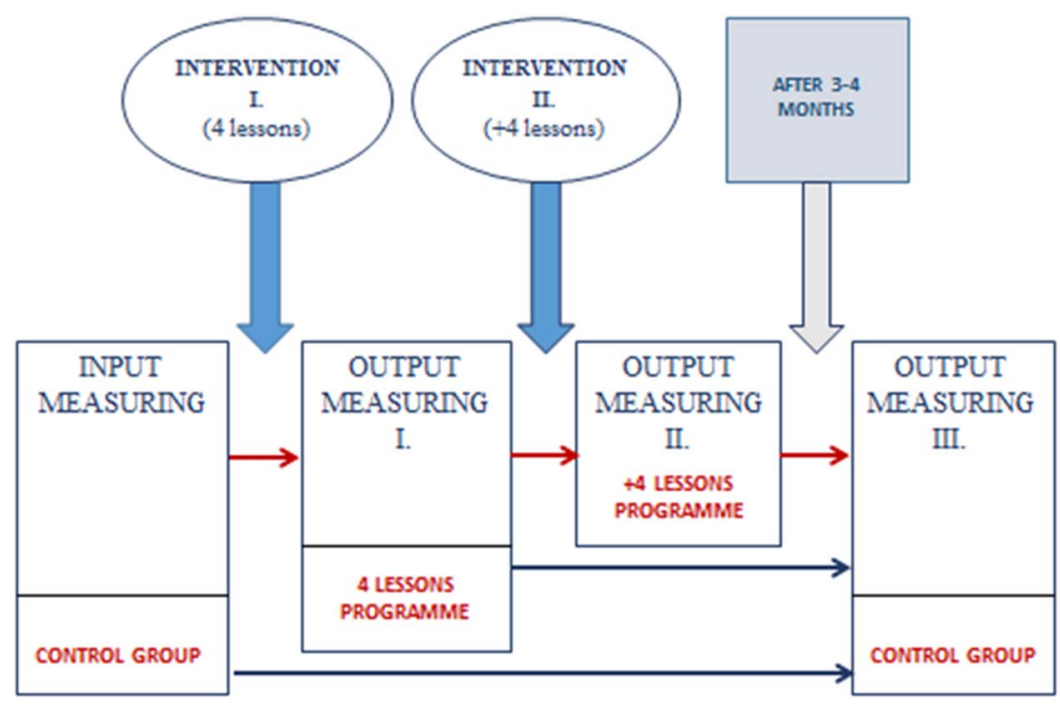

Figure 1. The research process of the Study, Teach, Understand Health Promotion Programme (STAnD Programme) 
programme has achieved its goals. We use the same questionnaire items (without measuring the programme satisfaction) and hand-rubbing technique assessment by UV-light-based imaging technology to compare the results of the different times (Figure 1).

After the last hand scanning (output measuring III.), we give an immediate feedback (demonstrated with the picture of the efficiency of handwashing) on the handwashing procedure for all students (including the control group). After 2 weeks, we retest the hand-rubbing techniques of the control group, so we are also able to test the effectiveness of the immediate feedback at the control group. This way, we can compare the effectiveness of the hand hygiene peer education training programme and of a visual demonstration at the same time.

\section{DISCUSSION AND CONCLUSIONS}

Hand hygiene is a fundamental part of everyday human culture. In order to improve the evidence base about the effect of hand hygiene intervention is one of the multiple aspects of environmental consciousness and represents a fundamental task in education.

Unfortunately, there are some biases such as underprivileged social and regional conditions, school absenteeism, as well as lacking the proper education in this topic, which can affect students' health in an unfavourable way. This is the reason why sociological health educational studies must be more rigorously designed, specifically addressing randomization, blind studies, fidelity of implementation, and attrition - all major sources of bias.

The purpose of this follow-up study was to demonstrate the extent to which the time and the number of repetitions of teaching influence the effectiveness of the teaching of handwashing procedures. Hand hygiene behaviour is an important element of our STAnD strategy.

In order to improve compliance (i.e., willingness to cooperate in our health education programme), one of the most important purposes is to use objective electronic technology capable for immediate feedback on the efficiency of the handwashing procedure. These tools are real-time devices providing immediate feedback to the person (e.g., student) about the handwashing, allowing one to change the "bad" habits that are already fixed. Improper set of movements during handwashing results in insufficient rubbing of the surface of the hand, for example, fingertips and at the inner base of thumbs.

Controlling and continuous training of the rubbing technique is just as important for effective training as maintaining high compliance. The effectiveness of peer education proceedings of proper handwashing was supported by an objective, scientific method using the Semmelweis Scanner tool developed to evaluate the rubbing technique. This electronic device has not been applied in peer education of hand hygiene to date.

The other important purpose of the STAnD Programme is to develop objective quantitative social research techniques. In this field, it is a complicated and complex challenge to find reliable and valid measurement instruments because of the low number of previous scientific measurements and the difficulties of evidence-based health promotion programmes.

We believe that, in turn, these well-perceived and computerized solutions, with quantitative social research techniques, are capable to sufficiently evaluate the effectiveness of hand hygiene trainings. In the course of the hand hygiene programme by means of both immediate and subsequent statistical analyses, we intend to demonstrate that an electronic device for direct feedback is suitable for increasing the motivation of students and to reduce the number of missed areas at the following handwashing.

Acknowledgements: The authors would like to thank HandinScan Zrt. and Ecolab-Hygiene Kft. for the personal support they provided our study.

Authors' contribution: HJF: wrote sections of "Introduction", "Sampling", "Research instruments of quantitative social research", and "Data collecting procedure". ÁLe: wrote "Measurement-based research" section, found references, and involved in checking of the manuscript. ÁLu: wrote sections of "Introduction" and "Research instruments of quantitative social research", found references, and involved in checking of the manuscript. EG: found references and involved in checking of the manuscript. RF: wrote "Measurement-based research" section and involved in checking of the manuscript. SDM, IKB, and ZSKS: involved in checking of the manuscript. ZSK: wrote "Data collecting procedure" section. AF: wrote sections of "Introduction", "Measurement-based research", "Data collecting procedure", and "Conclusion"; found references; involved in checking of the manuscript; and coordination of authors' contributions.

Ethical approval: Our research is morally acceptable and we follow the World Medical Association's Declaration of Helsinki and requirements of all applicable local and international standards. The Hungarian Medical Research Council Research Ethics Committee approved this research (no.18241-2/2017/EKU).

Funding: This study was funded by the Content Pedagogy Research Program of the Hungarian Academy of Sciences.

\section{REFERENCES}

1. Benè KL, Bergus G. When learners become teachers: a review of peer teaching in medical student education. Fam Med. 2014;46(10):783-87.

2. Parkin S, McKeganey N. The rise and the rise of peer education approaches. Drug-Educ Prev Policy. 2000;7(3):293-310.

3. Tolli MV. Effectiveness of peer education interventions for HIV prevention, adolescent pregnancy prevention and sexual health promotion for young people: a systematic review of European studies. Health Educ Res. 2012;27(5):904-13.

4. Southgate E, Aggleton P. Peer education: from enduring problematics to pedagogical potential. Health Educ J. 2016;76(1):1-12. 
5. Sloane BC, Zimmer CG. The power of peer health education. J Am Coll Health. 1993;41(6):241-45.

6. Turner G, Shepherd J. A method in search of a theory: peer education and health promotion. Health Educ Res. 1999;14(2):235-47.

7. Harden A, Oakley A, Oliver S. Peer-delivered health promotion for young people: a systematic review of different study designs. Health Educ J. 2001;60(4):339-53.

8. Feith HJ, Melicher D, Máthé G, et al. Experience and motivation: opinion of Hungarian high school students about health promotion programs. Orv Hetil. 2016;157(2):65-9.

9. Damon W. Peer education: the untapped potential. J Appl Dev Psychol. 1984;5(4):331-43.

10. Adamchak SE. Youth Peer Education in Reproductive Health and HIV/AIDS: Progress, Process, and Programming for the Future [Internet], Youth Issues Paper 7. Arlington: Family Health International, YouthNet Program; 2006 [cited 2017 Sep 11]. Available from: https://www. k4health.org/sites/default/files/Peer\%20Education\%20Over view.pdf

11. Sutherland EH, Cressey DR. Principles of Criminology. Philadelphia: Lippincott; 1960.

12. Sarbin TR, Allen VL, Rutherford EE. Social reinforcement, socialization, and chronic delinquency. Br J Soc Clin Psychol. 1965;4(3):179-84.

13. Bandura A. Self-efficacy: toward a unifying theory of behavioral change. Psychol Rev. 1977;84(2):191-215.

14. Klein NA, Sondag KA, Drolet JC. Understanding volunteer peer health educators' motivations: applying social learning theory. J Am Coll Health. 1994;43(3):126-30.

15. Guinan ME, McGuckin-Guinan M, Sevareid A. Who washes hands after using the bathroom? Am J Infect Control. 1997;25(5):424-25.

16. Guinan M, McGuckin M, Ali Y. The effect of a comprehensive handwashing program on absenteeism in elementary schools. Am J Infect Control. 2002;30(4):217-20.

17. Watson JA, Ensink JHJ, Ramos M, et al. Does targeting children with hygiene promotion messages work? The effect of handwashing promotion targeted at children, on diarrhoea, soil-transmitted helminth infections and behaviour change, in low- and middle-income countries. Trop Med Int Health. 2017;22(5):526-38.

18. Fewtrell L, Kaufmann RB, Kay D, et al. Water, sanitation, and hygiene interventions to reduce diarrhoea in less developed countries: a systematic review and meta-analysis. Lancet Infect Dis. 2005;5(1):42-52.

19. Schweizer ML, Reisinger HS, Ohl M, et al. Searching for an optimal hand hygiene bundle: a meta-analysis. Clin Infect Dis. 2014;58(2):248-59.

20. Chittleborough CR, Nicholson AL, Basker E, et al. Factors influencing hand washing behaviour in primary schools: process evaluation within a randomized controlled trial. Health Educ Res. 2012;27(6):1055-68.

21. Johansen A, Denbæk AM, Bonnesen CT, Due P. The Hi Five study: design of a school-based randomized trial to reduce infections and improve hygiene and well-being among 6-15 year olds in Denmark. BMC Public Health. 2015;15:207.

22. Kandel N, Lamichane J. Strategy of making hand washing a routine habit: principles of 5Es and 3Rs. JNMA J Nepal Med Assoc. 2016;55(203):40-4.

23. Early E, Battle K, Cantwell E, English J, Lavin JE, Larson E. Effect of several interventions on the frequency of handwashing among elementary public school children. Am J Infect Control. 1998;26(3):263-69.

24. HBSC.org [Internet]. Health Behavior in School-aged Children. St Andrews: WHO Collaborative Cross-National Survey; [cited 2017 Sep 11]. Available from: http://www.hbsc.org/methods/ index.html

25. Egeszseg.hu [Internet]. Health Communication Survey (HCS). Budapest, Hungary: National Institute for Health Development; [cited 2017 Sep 11]. Available from: http:// www.egeszseg.hu/szakmai_oldalak/oldal/szakmai-anyagok/ szakmai-anyagok-egeszsegkommunikacios-felmeres/

26. Szilágyi L, Haidegger T, Lehotsky Á, et al. A large-scale assessment of hand hygiene quality and the effectiveness of the WHO 6-steps. BMC Infect Dis. 2013;13:249.

27. Lehotsky Á, Szilágyi L, Demeter-Icl nzan A, Haidegger T, Wéber G. Education of hand rubbing technique to prospective medical staff, employing UV-based digital maging technology. Acta Microbiol Immunol Hung. 2016;63(2):217-28. 